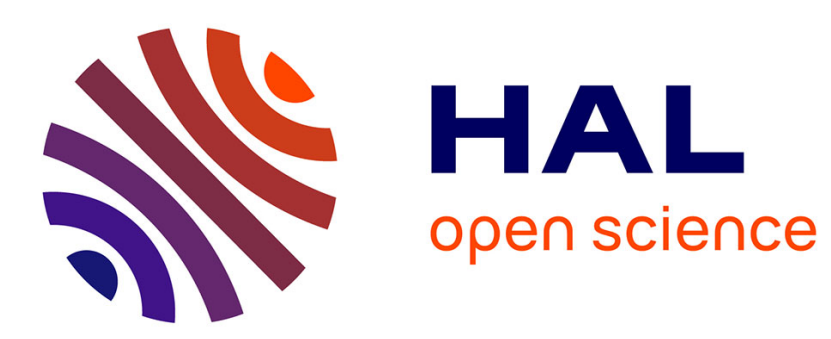

\title{
Stochastic modeling for hysteretic bit-rock interaction of a drill string under torsional vibrations
}

Fabio F Real, Anas Batou, Thiago Ritto, Christophe Desceliers

\section{To cite this version:}

Fabio F Real, Anas Batou, Thiago Ritto, Christophe Desceliers. Stochastic modeling for hysteretic bit-rock interaction of a drill string under torsional vibrations. Journal of Vibration and Control, 2019, pp.107754631982824. 10.1177/ToBeAssigned . hal-02050750

\section{HAL Id: hal-02050750 \\ https://hal.science/hal-02050750}

Submitted on 27 Feb 2019

HAL is a multi-disciplinary open access archive for the deposit and dissemination of scientific research documents, whether they are published or not. The documents may come from teaching and research institutions in France or abroad, or from public or private research centers.
L'archive ouverte pluridisciplinaire HAL, est destinée au dépôt et à la diffusion de documents scientifiques de niveau recherche, publiés ou non, émanant des établissements d'enseignement et de recherche français ou étrangers, des laboratoires publics ou privés. 


\title{
Stochastic modelling for hysteretic bit-rock interaction of a drill string under torsional vibrations
}

\author{
Fabio F Real ${ }^{1}$, Anas Batou², Thiago G Ritto ${ }^{3}$, Christophe Desceliers ${ }^{4}$
}

\begin{abstract}
This paper aims at constructing a stochastic model for the hysteretic behaviour of the nonlinear bit-rock interaction of a drill string under torsional vibrations using field data. The proposed model takes into account the fluctuations of the stick-slip oscillations and the hysteretic effect due to the nonlinear bit-rock interaction observed during the drilling process. These fluctuations are modelled by introducing a stochastic process associated with the variations of the bit-rock interaction during the drilling. The parameters of the stochastic model are calibrated with field data. Finally, independent trajectories of the proposed stochastic model are generated and used to simulate the stochastic response of the drill string torsional dynamics in presence of random bit-rock interaction. The stochastic model is then used to estimate statistics on the stability of the drill string.
\end{abstract}

\section{Keywords}

bit-rock stochastic interaction model, drillstring dynamics, hysteretic friction, experimental identification

\section{Introduction}

Drill string is a slender structure used for exploitation of oil reserves. A top drive rotates the system at the top, which transmits the torque to the bit that drills the rock. The design and analysis of drill strings are usually performed using a computational model. Several drill string models have been proposed in the literature. The complete dynamics of a drill string includes axial, torsional, and lateral vibrations Tucker RW and Wang C (1999); Ritto TG et al. (2009). In addition, in the development of directional drilling, advanced features taking into account the horizontal dynamics analysis might be necessary Ritto TG et al. (2013); Cunha A et al. (2015). Torsional control is also an issue Patil PA et al. (2013); Ritto TG and Ghandchi-Tehrani M (2018).

The present paper focuses on the analysis of the torsional vibration of the drill string, which is nonlinear due to the bitrock interaction. Field observations Pavone DR and Desplans JP (1994); Ritto TG et al. (2017); Real FF et al. (2018) show that the torque on bit varies nonlinearly with the bit speed. Furthermore, these observations show hysteretic cycles which fluctuate during the drilling process Real FF et al. (2018). Torsional vibration is a problem in drill string dynamics, especially when its length reaches thousands of meters. In some conditions, the lateral and axial vibrations are small, and the torsional vibration is the main concern Ritto TG et al. (2017); it might lead to stick-slip oscillations Kyllingstad A and Halsey GW (1988); Leine RI et al. (1998); Tucker RW and Wang C (1999); Richard T and Detournay E (2004); Khulief YA et al. (2007); Kreuzer E and Steidl M (2012); Patil PA et al. (2013); Hong L et al. (2010). In this severe conditions, the bit sticks (zero speed) then slips (high speed), and that might cause, for instance, measurement equipment failure, low rate of penetration, bit damage, and fatigue Wu X et al. (2012).
In Real FF et al. (2018), the authors have highlighted large fluctuations of the nonlinear bit-rock interaction law during the drilling. These fluctuations which are mainly due to the random soil mechanical properties during the drilling, and have to be taken into account in order to study the stability of the drill string with a good robustness. Objectives of the present paper are (1) to characterize and construct a probabilistic model of these fluctuations and (2) then to estimate the probability of instability of the drill string including the stochastic non-linear bit-rock interaction model. In the literature, only few papers are concerned with the probabilistic modelling of the bit-rock interaction. In Spanos PD et al. (2002), a stationary random process is considered to model lateral forces at the bit. In Ritto TG and Sampaio R (2012)), uncertainties are considered in the bit-rock interaction parameters. In Ritto TG et al. (2009), an adaptation of the nonparametric probabilistic approach Soize C (2000) is proposed to model globally the uncertainties in the bit-rock interaction model. For the two later referred papers, the probabilistic models are not time-dependent, i.e., the bit-rock interaction model is random but does not vary during the drilling. Constructing such a stochastic

\footnotetext{
${ }^{1}$ National Institute of Metrology, Quality and Technology - INMETRO, Brazil

2 School of Engineering, University of Liverpool, UK

3 Universidade Federal do Rio de Janeiro, Department of Mechanical Engineering, Brazil

4 Université Paris-Est, Laboratoire Modélisation et Simulation Multi Echelle, France
}

\section{Corresponding author:}

Thiago G Ritto, Universidade Federal do Rio de Janeiro Department of Mechanical Engineering, Ilha do Fundão, Rio de Janeiro, RJ, 21945-970, Brazil

Email: tritto@mecanica.ufrj.br 
computational models including the stochastic fluctuations of the bit-rock interaction forces would be helpful for robust optimization of the drill string Ritto TG et al. (2010).

In the present work, the nominal model of the drill string is constructed as two torsional beams which are discretized by means of the finite element method Jansen JD (1993); Khulief YA and Al-Naser H (2005); Ritto TG and Sampaio $\mathrm{R}$ (2012)), and a reduced-order model is constructed using the elastic modes. The non-linear bit-rock interaction forces are modelled as a point torque applied at the end of the bottom hole assembly (BHA). This simple model enables the efficient construction of the stability maps of the drill string. Then the stochastic non-linear bit-rock interaction model is constructed by introducing a multiplicative stationary Gaussian stochastic process. Field data are used to calibrate the power-spectral density function of this stochastic process. Once calibrated, independent realizations are generated and statistics on the drill string dynamical behaviour are estimated using the Monte Carlo simulation method. The results we obtain show that taking into account the stochastic fluctuations of the nonlinear bit-rock interaction enable an estimation of the probability of failure with respect to the stability of the drill string and offers robust method to design the drill string and its working condition (minimum top drive velocity for instance).

The paper is organized as follows. Section 2 describes the nominal drill string computational model, including the nonlinear bit-rock interaction. Section 3 presents the proposed probabilistic model for the bit-rock interaction, which is modelled as a nonstationary stochastic process to take into account its stochastic fluctuations during the drilling. The numerical results are presented in Section 4, where deterministic and stochastic dynamical response are analyzed. The last section brings the concluding remarks.

\section{Deterministic model for the drill string}

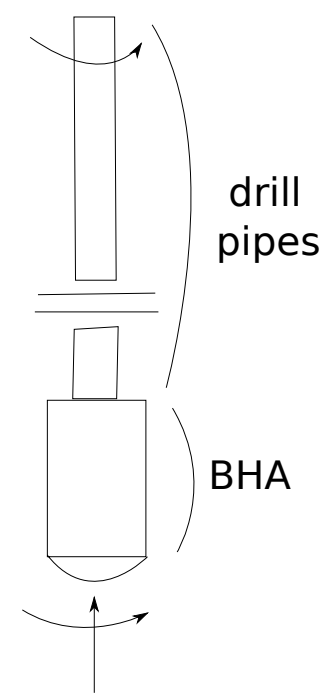

Figure 1. General scheme of a drill string.

Figure 1 presents a scheme of the torsional system considered in the analysis. A vertical wellbore, with only torsional vibrations, is taken into account. The system is composed of drill pipes (about 5,000 meters) and by the bottom hole assembly (BHA, about 400 meters). In the present paper lateral and axial vibrations are not modelled. The extension of the approach presented in this paper including lateral and axial vibrations is straightforward.

A constant angular speed $\Omega$ is imposed at the top and a reaction torque acts at the bottom due to the bit-rock interaction. In this paper, the torsional dynamics of the drill string is analysed by solving the torsional beam equation:

$$
\rho I_{p} \frac{\partial^{2} \boldsymbol{\theta}(x, t)}{\partial t^{2}}-G I_{p} \frac{\partial^{2} \boldsymbol{\theta}(x, t)}{\partial x^{2}}=\mathcal{T}(x, t),
$$

where $\boldsymbol{\theta}(x, t)$ is the angular rotation about the longitudinal $x$ axis related to time $(t), \mathcal{T}(x, t)$ is the torque per unit length, $I_{p}$ is the cross sectional polar area moment of inertia, and $\rho$ and $G$ are the mass density and shear modulus of the material of the column. The boundary conditions related to the imposed angular speed at the top are given by

$$
\left\{\begin{array}{c}
\boldsymbol{\theta}(0, t)=\Omega t \\
\dot{\boldsymbol{\theta}}(0, t)=\Omega
\end{array},\right.
$$

and the initial conditions are

$$
\boldsymbol{\theta}(x, 0)=0, \quad \dot{\boldsymbol{\theta}}(x, 0)=\Omega,
$$

As proposed in Real FF et al. (2018), the rotational displacements about a rotating frame is considered. Let $\boldsymbol{\theta}^{\text {rel }}(x, t)$ be the relative torsional degree of freedom in the rotating frame associated to the top sectional area (at $x=0$ ). We introduce the absolute rotational displacement as

$$
\boldsymbol{\theta}(x, t)=\Omega t+\boldsymbol{\theta}^{r e l}(x, t) .
$$

The system is discretized by means of the finite element model, where linear shape functions are applied. Let $\mathbf{u}(t)$ be the vector of $\boldsymbol{\theta}^{\text {rel }}(x, t)$ nodal values related to the drill string mesh. Note that, in the rotating frame, the angular displacement is fixed at the top because there is no relative displacement between the top drive and the first node, at the top of the drill string. Adding a proportional damping to the system, the vector $\mathbf{u}(t)$ is solution of the matrix equation

$$
[M] \ddot{\mathbf{u}}(t)+[D] \dot{\mathbf{u}}(t)+[K] \mathbf{u}(t)=\mathbf{T}(\dot{\mathbf{u}}(t), \ddot{\mathbf{u}}(t))),
$$

where $[M]$ is the mass matrix, $[D]$ is the damping matrix, $[K]$ is the stiffness matrix, and $\mathbf{T}(\dot{\mathbf{u}}(t), \ddot{\mathbf{u}}(t))$ is the torque vector. According to Eqs. (3) and (4), the initial conditions in the rotating frame read

$$
\mathbf{u}(0)=\mathbf{0}, \quad \dot{\mathbf{u}}(0)=-\Omega \mathbf{1} .
$$

where $\mathbf{1}=\left[I_{n \times 1}\right]$ a vector with all entries equal to one. All the components of the torque vector are zero except the one corresponding to the drill bit node (corresponding to $x=L)$. For this node, the nonlinear torque applied to the bit is denoted by $T_{\text {bit }}\left(\dot{\theta}_{\text {bit }}(t), \ddot{\theta}_{\text {bit }}(t)\right)$ and will be described in the next section.

The normal modes of the conservative homogeneous system are used to construct a reduced-order model. The $m$ first eigenvalues $0<\lambda_{1} \leq \lambda_{2} \leq \ldots \leq \lambda_{m}$ associated with elastic modes $\left\{\boldsymbol{\varphi}_{1}, \boldsymbol{\varphi}_{2}, \ldots, \boldsymbol{\varphi}_{m}\right\}$ are solutions of the generalized eigenvalue problem

$$
[K] \varphi=\lambda[M] \varphi
$$


The reduced-order model is obtained by projecting the full computational model on the subspace spanned by the $m$ first elastic modes calculated using Eq. (7). Let $[\Phi]$ be $n \times m$ matrix whose columns are the $m$ first elastic modes. We can then introduce the following approximation

$$
\mathbf{u}(t)=[\Phi] \mathbf{q},
$$

in which $\mathbf{q}$ is the vector of the $m$ generalized coordinates which are solution of the reduced matrix equation

$$
[\widetilde{M}] \ddot{\mathbf{q}}(t)+[\widetilde{D}] \dot{\mathbf{q}}(t)+[\widetilde{K}] \mathbf{q}(t)=\tilde{\mathbf{T}}(\dot{\mathbf{q}}(t), \ddot{\mathbf{q}}(t)),
$$

with the initial conditions

$$
\mathbf{q}(0)=\mathbf{0}, \quad \dot{\mathbf{q}}(0)=-\Omega[\widetilde{M}]^{-1}[\Phi]^{T}[M] \mathbf{1} .
$$

In these equations, $[\widetilde{M}]=[\Phi]^{T}[M][\Phi],[\widetilde{D}]=[\Phi]^{T}[D][\Phi]$ and $[\widetilde{K}]=[\Phi]^{T}[K][\Phi]$ are $m \times m$ mass, damping and stiffness reduced-order matrices, and $\tilde{\mathbf{T}}(\dot{\mathbf{q}}(t), \ddot{\mathbf{q}}(t))=$ $\left.[\Phi]^{T} \mathbf{T}([\Phi] \dot{\mathbf{q}}(t), \Phi] \ddot{\mathbf{q}}(t)\right)$ is the vector of the reduced-order generalized torque. The set of equations (8), (9) and (10) can be solved using commonly used integration schemes, such as the Euler scheme or the Runge-Kutta, for instance.

\section{Probabilistic model for the bit-rock interaction with hysteretic effects}

Figure 2 shows the field data Ritto TG et al. (2017); Real FF et al. (2018) that will support the proposed model presented in this section. The downhole information used in this paper was acquired using a downhole mechanics measurement unit capable of providing both real-time measurement through mud telemetry and continuously recorded high-frequency data throughout the run. The sub, installed at the BHA above the bit, contains a suite of 19 sensors sampled at $10,000 \mathrm{~Hz}$ and downsampled and filtered prior to recording at $50 \mathrm{~Hz}$. Following is a list of $50-\mathrm{Hz}$ data recorded in this sub: triaxial accelerations; gyro rpm; magnetometer rpm; axial loading; torque; bending moment Shi J et al. (2016).

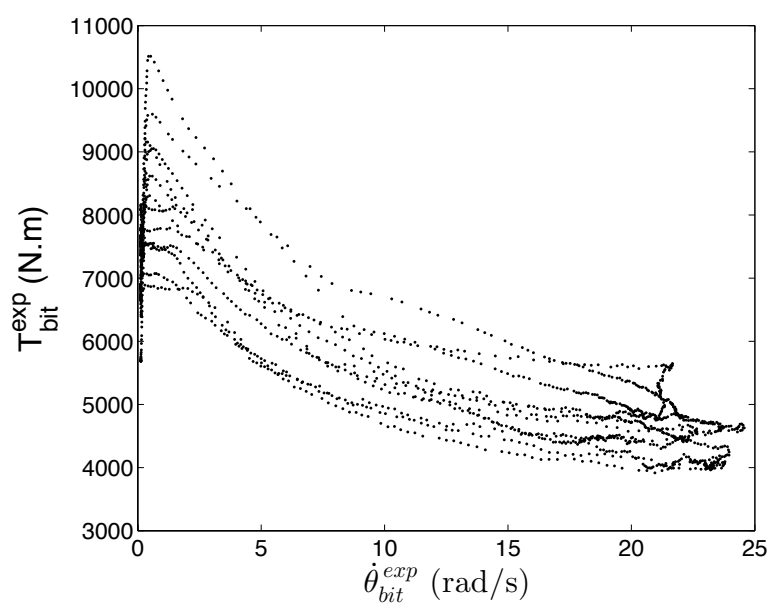

Figure 2. Field data stick-slip cycles: measured torque $T_{\mathrm{bit}}^{\exp }$ versus the bit speed $\dot{\theta}_{\mathrm{bit}}$.

The field data shown in Fig. 2 was measured at the BHA, very close to the drill bit. A window average smoothing was used to filter measurements noise and obtain these stickslip cycles. A deterministic bit-rock interaction model that takes into account the observed hysteresis (non-reversibility) effect was proposed in Real FF et al. (2018). Hysteretic (non-reversible) behaviour is more evident when the drill bit is slipping (high torsional oscillations). This macroscopic phenomenon is related to a frictional memory due a friction force's delay, where the bandwidth of the loop increases according to the drill string acceleration Hess DP and Soom A (1990); Olsson KJ et al. (1998); Wojewoda J et al. (2008). The deterministic hysteretic bit-rock interaction model considered in the present paper Real FF et al. (2018) reads

$\underline{T}_{\mathrm{bit}}\left(\dot{\theta}_{\mathrm{bit}}, \ddot{\theta}_{\mathrm{bit}}\right)=b_{0}\left(\tanh \left(b_{1} \dot{\theta}_{\mathrm{bit}}\right)+\frac{b_{2} \dot{\theta}_{\mathrm{bit}}^{b_{4}}}{1+b_{3} \dot{\theta}_{\mathrm{bit}}^{b_{5}}}\left(1+H\left(\dot{\theta}_{\mathrm{bit}} \ddot{\theta}_{\mathrm{bit}}\right)\right),\right)$,

for $\dot{\theta}_{\mathrm{bit}}>0$, in which $b_{0}, b_{1}, b_{2}, b_{3}, b_{4}, b_{5}$ are positive parameters to be fitted such that $0<b_{4}<b_{5}$. The bit-rock interaction model Eq. (11) Real FF et al. (2018) contains a hysteretic function $H$, which is a function of the bit angular acceleration (to separate forward and backward phases) and which is defined by

$$
H\left(\dot{\theta}_{\mathrm{bit}}, \ddot{\theta}_{\mathrm{bit}}\right)=\beta_{1} \tanh \left(\beta_{2} \ddot{\theta}_{\mathrm{bit}}\right),
$$

where $\beta_{1}$ and $\beta_{2}$ are two positive parameters. Note that the hysteretic cycle is limited $\left(1 \pm \beta_{1}\right)$, and if $H$ equals to zero there is no hysteresis.

In Real FF et al. (2018), the parameters of this bitrock interaction model have been calibrated such that it fits with the mean field data cycle. The optimal parameters are $b_{0}=-3478, b_{1}=938, b_{2}=2.56, b_{3}=0.38, b_{4}=0.78$, and $b_{5}=1.1, \beta_{1}=14 \%, \beta_{2}=10.6$.

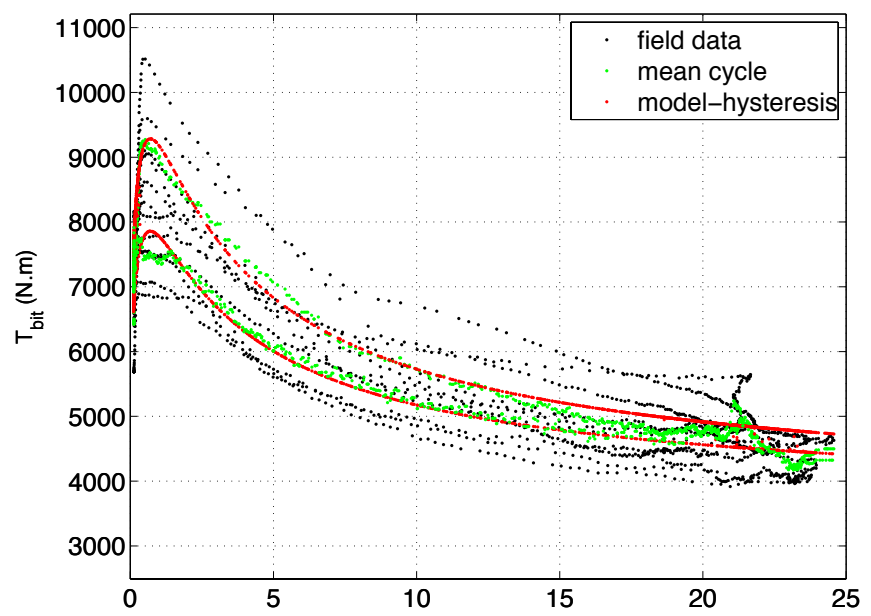

Figure 3. Bit-rock interaction: field data (black), mean field data cycle (green) and bit-rock interaction model with hysteresis (red).

Figure 3 compares the field data with the deterministic model. It can be seen that, in average, the bit-rock interaction model, Eq.(11), presents a reasonable good fit comparing to the field data mean cycle. Nevertheless, it is noticed a considerable time fluctuation of the field data cycles. This fluctuation is mainly due to the variation of the soil mechanical properties during the drilling (the 
measurement noise has already been filtered using a window averaging). The probabilistic modelling of this fluctuation is the objective of this paper.

If we take a close look at the field data, we notice that the torque on bit varies from about 7 to $10.5 \mathrm{kNm}$, when the bit speed is close to $1 \mathrm{rad} / \mathrm{s}$. For higher speeds, 20 $\mathrm{rad} / \mathrm{s}$, the torque on bit varies from about 4 to $5.8 \mathrm{kNm}$. The distance between the higher and lower values is very different, depending on the bit speed: 3.5 and $1.8 \mathrm{kNm}$. But, for both speeds the torque on bit is varying plus or minus $20 \%$. Therefore, the present paper proposes to take into account the stochastic fluctuations of the bit-rock interaction model by including a multiplicative stochastic process to the deterministic model:

$$
T_{\mathrm{bit}}\left(\dot{\theta}_{\mathrm{bit}}(t), \ddot{\theta}_{\mathrm{bit}}(t)\right)=\underline{T}_{\mathrm{bit}}\left(\dot{\theta}_{\mathrm{bit}}(t), \ddot{\theta}_{\mathrm{bit}}(t)\right)(1+\eta(t)),
$$

where $\eta(t)$ is a centred stochastic process $\eta(t)$ which can be rewritten as

$$
\eta(t)=\frac{T_{\mathrm{bit}}\left(\dot{\theta}_{\mathrm{bit}}(t), \ddot{\theta}_{\mathrm{bit}}(t)\right)}{\underline{T}_{\mathrm{bit}}\left(\dot{\theta}_{\mathrm{bit}}(t), \ddot{\theta}_{\mathrm{bit}}(t)\right)}-1 .
$$

The experimental stochastic process $\eta^{\exp }(t)$ can be computed using Eq. (14) and the field data related to the torque on bit. This stochastic process is shown in Fig. 4. Unfortunately, there is not enough field data to completely characterize this stochastic process. We will assume here that $\eta(t)$ is a centred stationary Gaussian stochastic process. This assumption will be verified in future work with more experimental data. The power spectral density (PSD) is estimated using the periodogram method Priestley MB (1981). Figure 5 shows the estimated field data PSD.

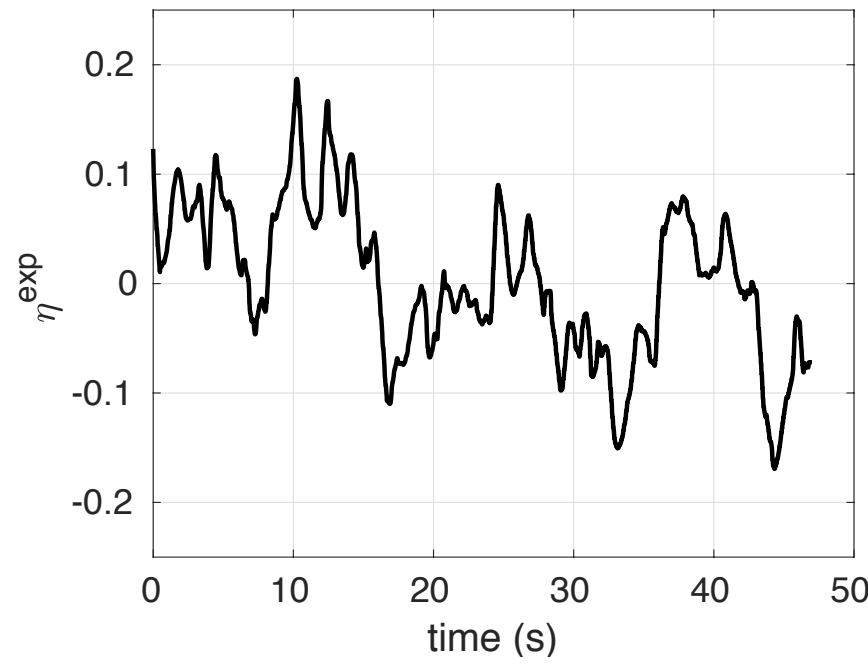

Figure 4. Stochastic process $\eta^{\exp }(t)$ obtained experimentally with field data.

Regarding Figure 5, the PSD is constant until a critical frequency and then decreases linearly (in log-log scale). This type of PSD is often encountered when addressing turbulent forces Batou A and Soize C (2009). Then the proposed PSD model $S(f)$ is written as

$$
\begin{aligned}
\log (S(f))=A_{0} & \text { for } \quad \mathrm{f}<\mathrm{f}_{0}, \\
\log (S(f))=a \log (f)+b & \text { for } \quad \mathrm{f} \geq \mathrm{f}_{0},
\end{aligned}
$$

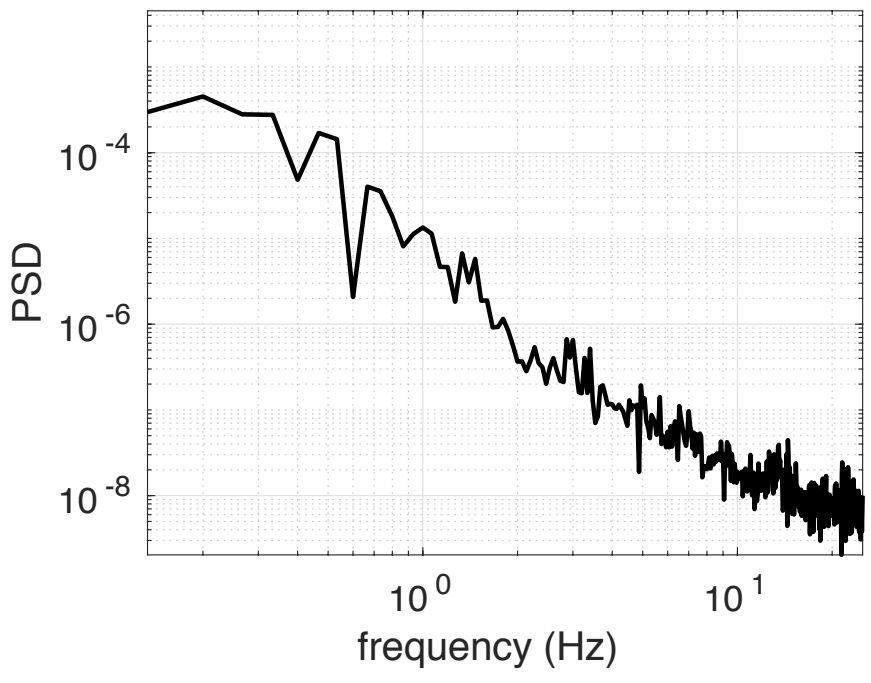

Figure 5. Field data PSD.

where $f_{0}, A_{0}, a$ and $b$ are the parameters of the model. These parameters are calibrated using the experimental PSD such that $f_{0}=0.27, A_{0}=-7.6, a=-3.13$ and $b=-11.67$ (with appropriate units). Figure 6 compares the calibrated PSD model with field data PSD, where a reasonable agreement is observed.

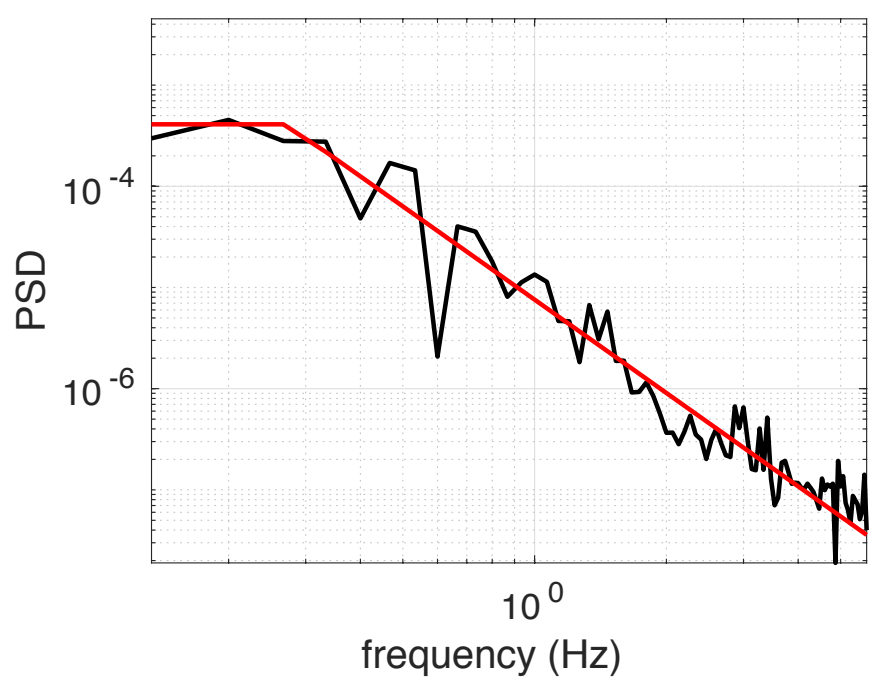

Figure 6. Comparison between the calibrated PSD and the field data PSD.

With the PSD $S(f)$ in hands one can generate independent realizations of the stochastic process $\eta(t)$ using a classical generator of Gaussian process Benaroya (2005). Figure 7 shows two independent trajectories of $\eta(t)$, which again give reasonable agreement with the observed process $\eta^{\exp }(t)$ shown in Figure 4.

\section{Simulation of the stochastic drill string dynamics}

\subsection{Analysis of one realization of the stochastic drill string dynamical response}

The previous section was concerned with the construction of a stochastic bit-rock interaction model including hysteretic cycles fluctuations. A stationary stochastic process was 

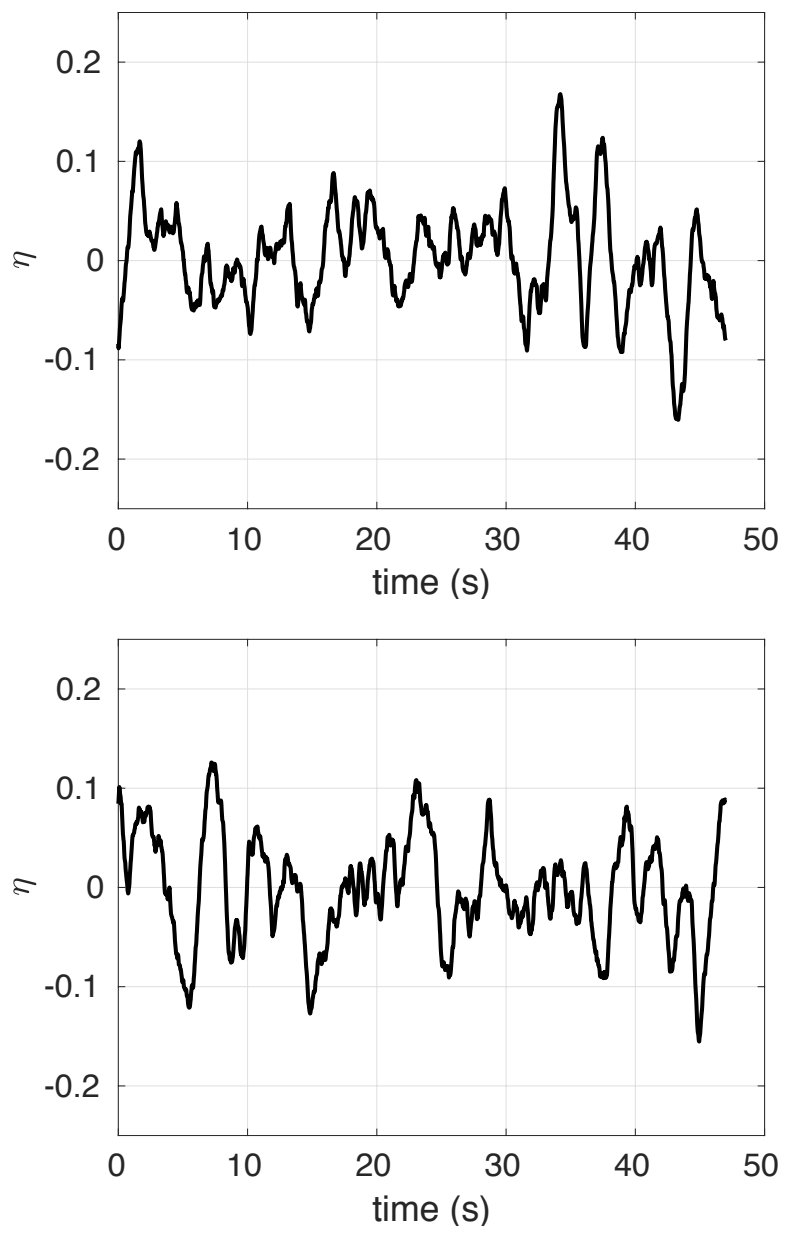

Figure 7. Two independent realizations of the stochastic process $\eta(t)$.

introduced for the computation of the torque on bit, Eq.(13). The stochastic bit-rock interaction model is then added to the torsional drill string model, Eq.(9), and the stochastic non-linear dynamical response of the drill string is computed and analysed. Note that Eq.(9) becomes random because of the random bit-rock interaction, Eq.(13). The general integration scheme for one realization of the stochastic bitrock interaction model is presented in Algorithm 1.

Table 1 contains the parameters of the drill string used for the simulation.

The mass and stiffness matrices are constructed using 100 finite elements, after convergence check. The generalized damping matrix is diagonal with damping ratios equal to 0.005 for the first mode, 0.03 for the second and third modes, and 0.005 for all the other modes. The first five natural frequencies computed for the system are: $0.13,0.42,0.74$, 1.07, $1.41 \mathrm{~Hz}$.

The non-linear equation (9) is solved using a modified Euler scheme with a time step $0.512 \mathrm{~ms}$. For one realization of the stochastic bit-rock interaction model, Figure 8 shows the stochastic response of the drill string in the stationary regime. In comparison with Figure 2, it can be observed the same stochastic behaviour but with a slightly larger value of the maximum angular velocity. This difference is due to the Finite Element model used here which can not reproduce all the complexity of a real drill string dynamics. But the objective of the paper concerns the construction

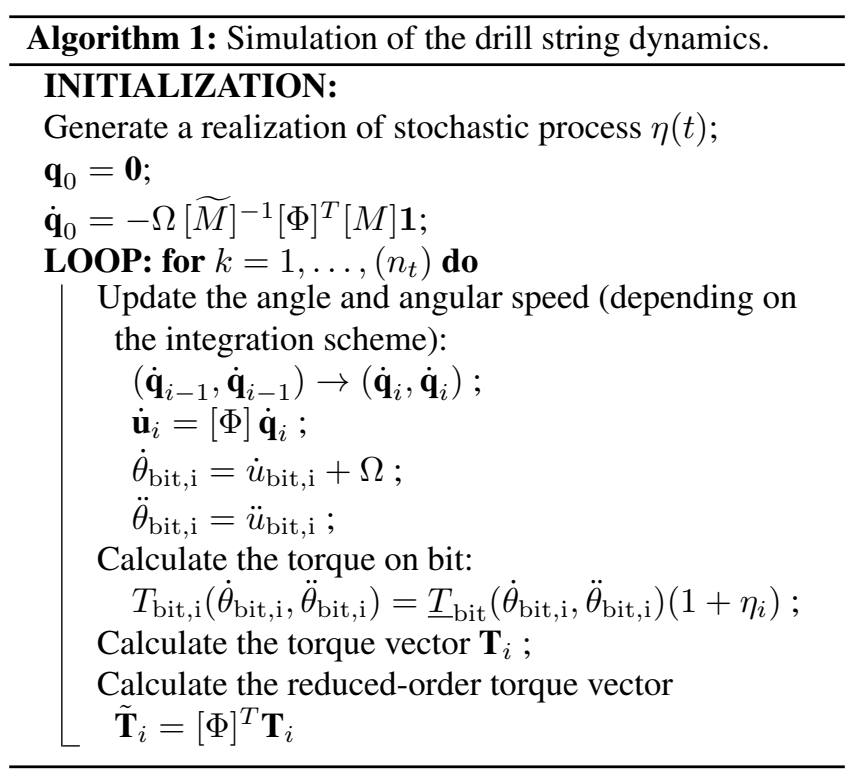

\begin{tabular}{|l|c|c|}
\hline & DP & BHA \\
\hline Elastic Modulus [GPa] & 220 & 220 \\
\hline Poisson's coefficient & 0.29 & 0.29 \\
\hline Volumetric mass density $\left[\mathrm{kg} / \mathrm{m}^{3}\right]$ & 7,800 & 7,800 \\
\hline Length [m] & $4,733.60$ & 466.45 \\
\hline Inner radius [m] & 0.0595 & 0.0363 \\
\hline Outer radius [m] & 0.070 & 0.0803 \\
\hline
\end{tabular}

Table 1. Drill string characteristics.

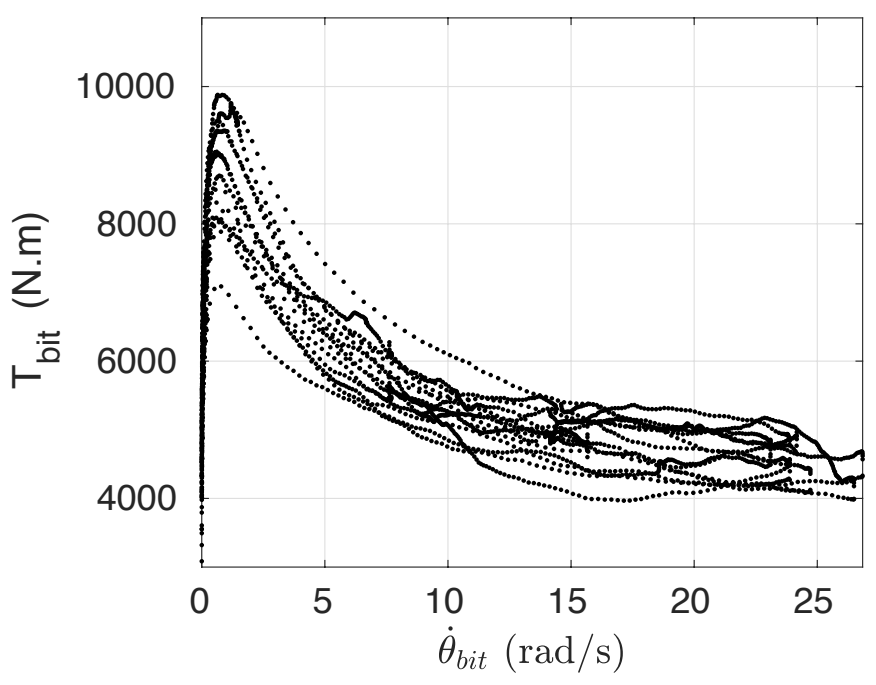

Figure 8. One realization of the simulated bit-rock interaction.

of the stochastic bit rock interaction model which should not depend on the computational model of the drill string. Figure 9 compares the steady-state of random bit response using the proposed stochastic bit-rock interaction model with the corresponding time range response obtained using the deterministic model described by Eq. (11). We are interested in range While a 4-cycles periodic regime is reached for the deterministic case, as expected, no periodicity is observed in the stochastic case.

For the deterministic case, the 4-cycles periodic sequence for the duration of the stick and slip phases are respectively $(1.34,2.53,2.48) \mathrm{s}$ and $(5.4,6.28,6.41) \mathrm{s}$. For the stochastic 

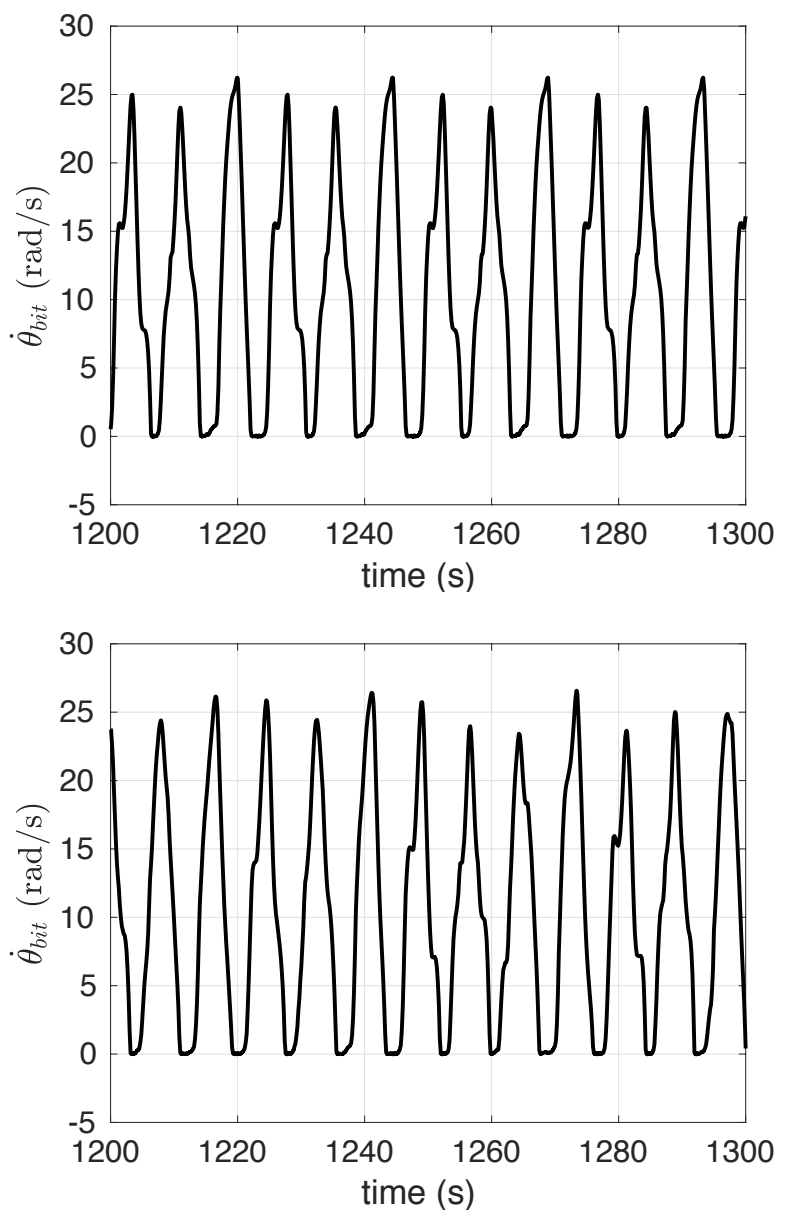

Figure 9. Steady-state of bit angular velocity: deterministic bit-rock interaction model (top, 4-cycles periodic regime is observed) and stochastic bit-rock interaction model (bottom, no periodicity is observed) $-5,250 \mathrm{~s}$ of total simulation time.

case, these durations are random and their associated probability distribution (obtained statistically using 750 cycles) are represented on Fig. 10. We can see is these figures a large variability of the stick and slip durations with modes closed to values corresponding to the values calculated with a deterministic bit-rock interaction model.

\subsection{Stochastic stability analysis}

Now the analysis is extended to quantify statistics on the stability threshold of the system, as the imposed speed at the top varies. The torsional stability of a drill string can be quantified through the stick-slip severity factor, defined by Ritto TG et al. (2017)

$$
\gamma_{\mathrm{SS}}(\Omega)=\frac{\dot{\theta}_{\mathrm{bit}}^{\max }(\Omega)-\dot{\theta}_{\mathrm{bit}}^{\min }(\Omega)}{2 \Omega},
$$

where $\dot{\theta}_{\mathrm{bit}}^{\max }(\Omega)$ and $\dot{\theta}_{\mathrm{bit}}^{\min }(\Omega)$ are the minimum and maximum bit velocity in the steady-state regime. In case of pure slipping, $\gamma_{\mathrm{SS}}=0$. If there are stick-slip oscillations then $\gamma_{\mathrm{SS}}>0$.

First, the deterministic system is analyzed. The stick-slip severity factor in the range $B=[6 ; 27] \mathrm{rad} / \mathrm{s}$ is plotted in Fig. 11. As expected, the stick-slip severity factor decreases when the imposed rotation at the top increases. If the speed at the top is lower than $16 \mathrm{rad} / \mathrm{s}, \gamma_{\mathrm{SS}}$ is greater than 0.5 .
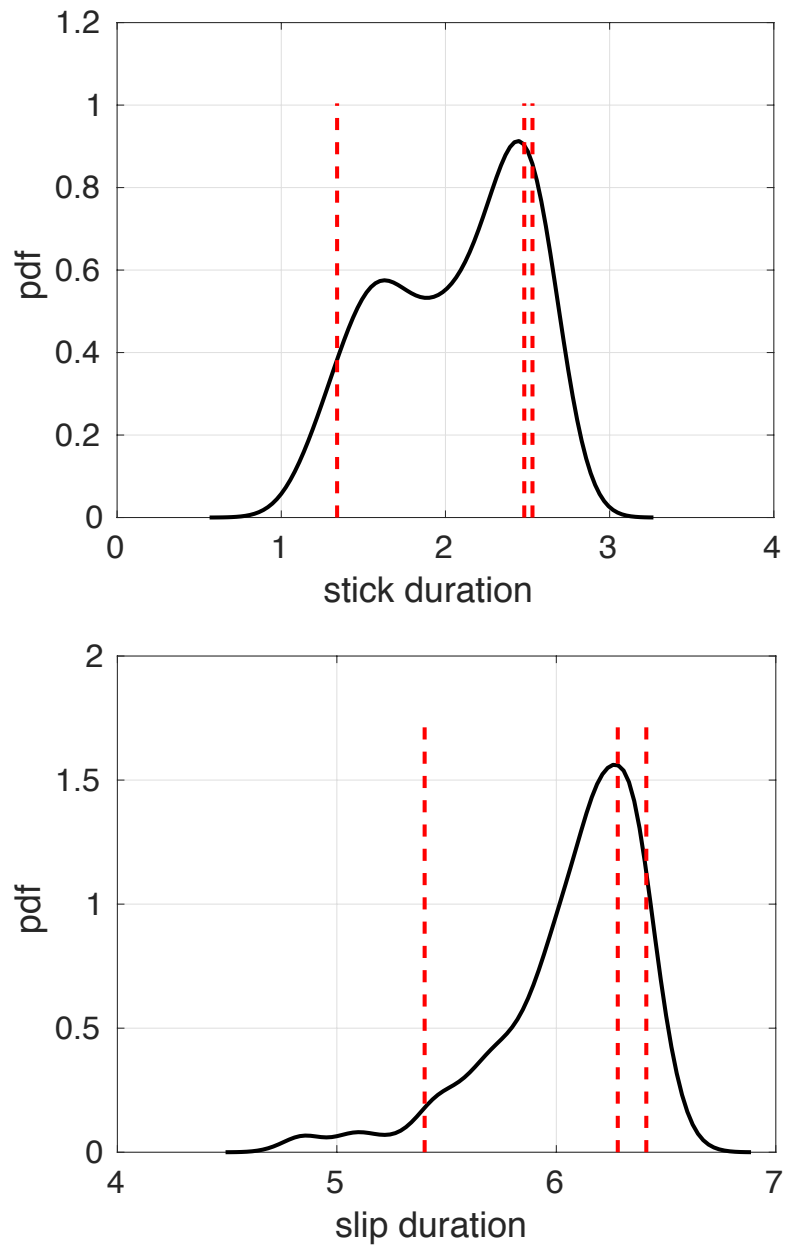

Figure 10. Random stick and slip phases duration: probability density functions (black line) and corresponding 4-cycles periodic values calculated with a deterministic bit-rock interaction model (red vertical lines).

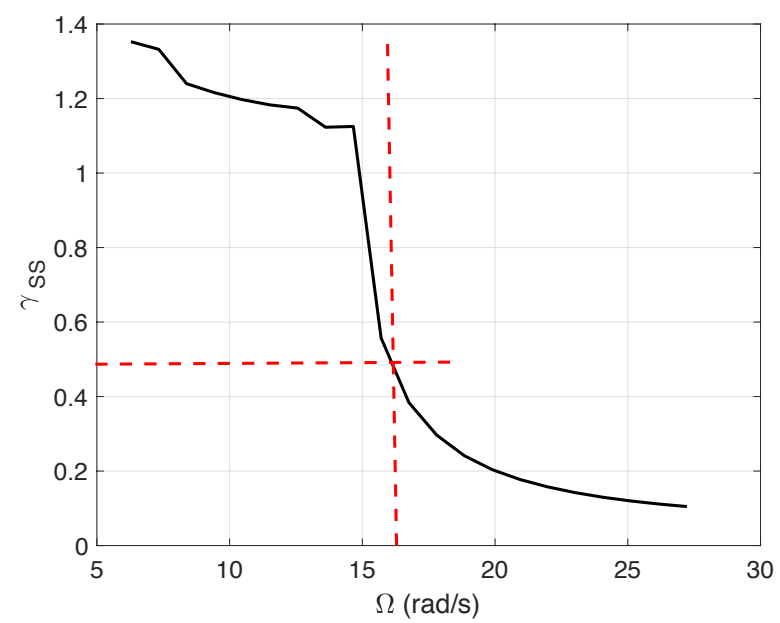

Figure 11. Stick-slip severity factor for a deterministic bit-rock interaction. Red dashed line indicates the stability threshold of $\gamma_{\mathrm{SS}}$.

For the stochastic bit-rock interaction model, the stickslip severity factor becomes random and its statistics are estimated using the Monte Carlo simulation method with $n_{s}=500$ samplings. For each Monte Carlo loop, an independent realization of the stochastic bit-rock interaction 
model is generated, a realization of the stochastic angular velocity is calculated and the corresponding stick-slip severity factor is determined on the stationary regime. Figure 13 shows statistics on the random stick-slip severity factor. The convergence with respect to the number of samplings $n_{s}$ is analysed by introducing the convergence function

$$
C V\left(n_{s}\right)=\frac{1}{n_{s}} \sum_{i=1}^{n_{s}} \int_{B} \gamma_{\mathrm{SS} i}(\omega)^{2} d \omega,
$$

where $\gamma_{\mathrm{SS} i}$ corresponds to the $i^{\text {th }}$ calculated realization of the stick-slip severity factor, and $B$ is the integration domain. The convergence function which is plotted in Figure 12. We can see in this figure that a good convergence is achieved using 500 realizations.

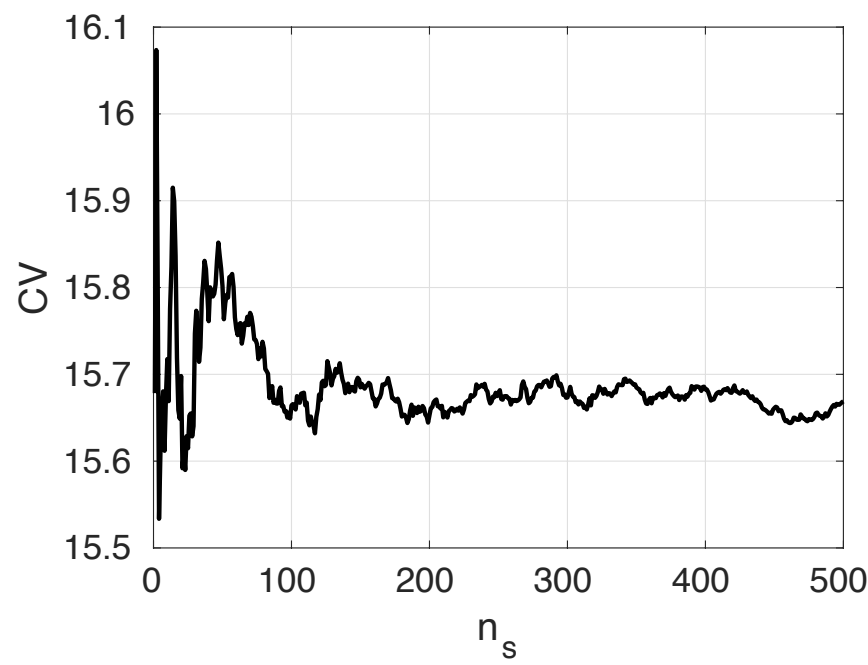

Figure 12. Convergence function $n_{s} \mapsto C V\left(n_{s}\right)$.

The statistical envelope shown in Fig. 13, due to the stochastic bit-rock interaction, yields large fluctuation in the random stick-slip severity factor. This means that bit-rock interaction variability has a direct impact on the drill string stability and should therefore be taken into account for a robust analysis of the drill string dynamics. The results show that when the top speed is about $20.5 \mathrm{rad} / \mathrm{s}$ the system has $5 \%$ probability of having the value of $\gamma_{\mathrm{SS}}$ greater than 0.5 . The probability of instability increases as the speed decreases. This result brings much more information comparing with the deterministic result, where, for the same threshold of $\gamma_{\mathrm{SS}}<0.5$, the limit speed of $16 \mathrm{rad} / \mathrm{s}$ was obtained.

\section{Concluding Remarks}

In the present paper, a new probabilistic model for the bitrock interaction model is proposed. This model includes a multiplicative stochastic process to take into account fluctuations of the torque on bit during the drilling. The stochastic model was calibrated with field data, and it takes into account hysteretic cycles and their stochastic fluctuations. The proposed bit-rock probabilistic model can be constructed independently from the computational model of the column.

The deterministic and stochastic torsional dynamics of a drill string are analyzed and a reasonable agreement between model predictions and field data is observed. The statistics of the stick and slip duration were also analyzed.

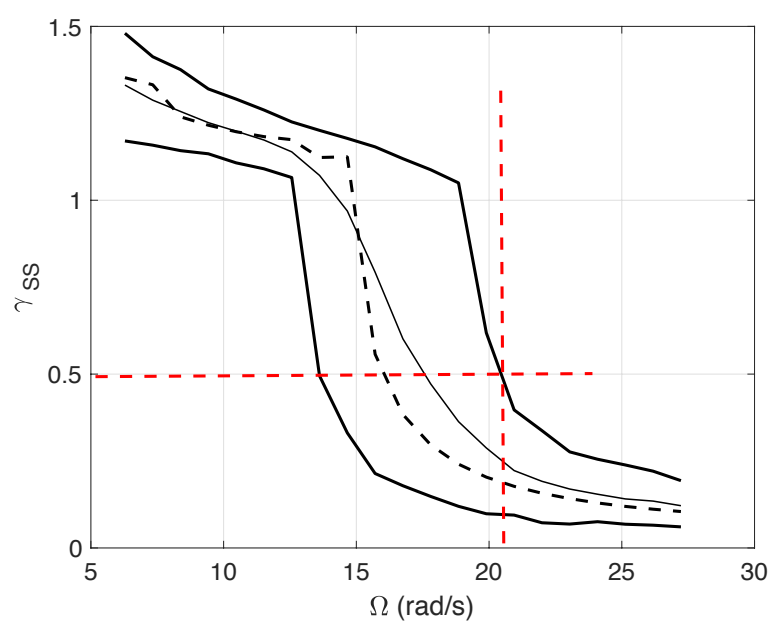

Figure 13. Random stick-slip severity factor for a stochastic bit-rock interaction. Solid lines: mean response and $90 \%$ confidence region. Dashed line: deterministic case (black), stability threshold (red).

A considerable impact of the proposed stochastic model on the torsional stability of the system was observed. For this end, the stick-slip severity factor is computed and statistical envelopes are plotted for varying imposed speed at the top. A robust estimation of the minimum angular speed is estimated using these plots.

In future works, additional experimental data will be collected in order to validate our assumptions on the probability distribution of the stochastic process $\eta(t)$ introduced in the stochastic non-linear bit rock interaction model. Also, the PSD is likely to depend on the imposed angular speed. The future experimental results will enable the characterization of this angular speed dependency.

\section{Conflict of interest}

The authors declare no conflict of interest in preparing this article

\section{Acknowledgements}

The authors are grateful to the financial support of the Brazilian agencies CAPES, CNPq, and FAPERJ, as well the "Agence Nationale de la Recherche" (Contract HiMoDe, ANR-12-JS09-0014).

\section{References}

Batou A, Soize C, (2009) Experimental identification of turbulent fluid forces applied to fuel assemblies using an uncertain model and fretting-wear estimation. Mechanical Systems and Signal Processing 23(7):21412153.

Benaroya H, Han SM (2005) Probability Models in Engineering and Science. Taylor and Francis.

Cunha A, Soize C, Sampaio R (2015) Computational modeling of the nonlinear stochastic dynamics of horizontal drillstrings. Computational Mechanics 56(5):849-878. 
Hess DP, Soom A (1990) Friction at lubricated line contact operating at oscillating sliding velocities. Journal of Tribology 112:147-152.

Hong L, Girsang IP, Dhupia JS (2010) Identification and control of stick-slip vibrations using Kalman estimator in oil-well drill strings. Journal of Petroleum Science and Engineering 140:119-127.

Jansen JD (1993) Nonlinear dynamics of oilwell drill strings. PhD thesis, Technische Universiteit Delft.

Khulief YA, Al-Naser H (2005) Finite element dynamic analysis of drillstrings. Finite Elements in Analysis and Design, 41:1270-1288.

Khulief YA, Al-Sulaiman FA, Bashmal S (2007) Vibration analysis of drillstrings with self-excited stick-slip oscillations. Journal of Sound and Vibration 299(3):540558.

Kyllingstad A, Halsey GW (1988) Study of slip/stick motion of the bit. SPE drilling engineering 3(4):369-373.

Kreuzer E, Steidl M (2012) Controlling torsional vibrations of drill strings via decomposition of traveling waves. Archive of Applied Mechanics 82(4):515-531.

Leine RI, Van Campen DH, van den Steen L (1998) Stickslip vibrations induced by alternate friction models. Nonlinear Dynamics 16:41-54.

Olsson H, Astrom KJ, Canudas de Wit C, Gafvert M, Lischinsky P (1998), Friction models and friction compensation. European Journal of Control 4(3):176195.

Patil PA, Teodoriu C (2013) A comparative review of modelling and controlling torsional vibrations and experimentation using laboratory setups. Journal of Petroleum Science and Engineering 112:227-238.

Pavone DR, Desplans JP (1994) Application of high sampling rate downhole measurements for analysis and cure of stick/slip in drilling. Proceedings - SPE Annual Technical Conference and Exhibition 335-345.

Priestley MB (1981) Spectral Analysis and Time Series. Academic Press, New York.

Real FF, Batou A, Ritto TG, Desceliers C, Aguiar RR (2018) Hysteretic Bit/Rock Interaction Model to Analyze the Torsional Dynamics of a Drill String. Mechanical System and Signal Processing, 111:222-233.

Richard T, Detournay E (2004) Self-excited stick-slip vibrations of drill bits. Comptes Rendus de Mecanique (332):619-626.

Ritto TG, Soize C, Sampaio R (2009) Nonlinear dynamics of a drill-string with uncertain model of the bitrock interaction. International Journal of NonLinear Mechanics 44(8):865-876.

Ritto TG, Soize C, Sampaio R (2010) Robust optimization of the rate of penetration of a drill-string using a stochastic nonlinear dynamical model. International Computational Mechanics 45(50):415-427.

Ritto TG, Sampaio R (2012) Stochastic drill-string with uncertainty on the imposed speed and on the bitrock parameters. International Journal for Uncertainty Quantification 2(2):111-124.

Ritto TG, Escalante MR, Sampaio R, Rosales MB (2013) Drill-string horizontal dynamics with uncertainty on the frictional force. Journal of Sound and Vibration 332(1):145-153.

Ritto TG, Aguiar RR, Baieb SH (2017) Validation of a drill string dynamical model and torsional stability. Meccanica 52(11-12):2959-2967.

Ritto TG, Ghandchi-Tehrani M (2018) Active control of stick-slip torsional vibrations in drill-strings, Journal of Vibration and Control, article accepted for publication on April 9th 2018.

Shi J, Durairajan B, Harmer R, Chen W, Verano F, Arevalo Y, Douglas C, Turner T. Trahan D, Touchet J, Shen Y, Zaheer A, Pereda F, Robichaux K, Cisneros D (2016), Integrated Efforts to Understand and Solve Challenges in 26-in Salt Drilling, Gulf of Mexico. SPE 180349-MS , SPE Deepwater Drilling \& Completions Conference, Galveston, Texas, USA.

Soize C (2000) A nonparametric model of random uncertainties for reduced matrix models in structural dynamics. Probabilistic Engineering Mechanics 15(3):277-294.

Spanos PD, Chevallier AM, Politis NP (2002) Nonlinear stochastic drill-string vibrations. Journal of Vibration and Acoustics. Transactions of the ASME 124(4):512518.

Tucker RW, Wang C (1999) On the effective control of torsional vibrations in drilling systems. Journal of Sound and Vibration 224(1):101-122.

Wojewoda J, Stefanski A, Wiercigroch M, Kapitaniak T (2008) Hysteretic effects of dry friction: modelling and experimental studies. Philosophical Transactions of the Royal Society A: Mathematical, Physical and Engineering Sciences 366(1866):747-765.

Wu X, Karuppiah V, Nagaraj M, Partin UT, Machado M, Franco M, Duvvuru HK (2012) Identifying the Root Cause of Drilling Vibration and Stick-Slip Enables Fit-for-Purpose. IADC/SPE Drilling Conference and Exhibition, no. 151347. 